\title{
Lymph node metastasis in thymic malignancies: A Chinese multicenter prospective observational study
}

\author{
Wentao Fang, MD, ${ }^{\mathrm{a}}$ Yun Wang, MD, ${ }^{\mathrm{b}}$ Liewen Pang, $\mathrm{MD},{ }^{\mathrm{c}}$ Zhitao Gu, MD, ${ }^{\mathrm{a}}$ Yucheng Wei, MD, ${ }^{\mathrm{d}}$ \\ Yongyu Liu, MD, ${ }^{\mathrm{e}}$ Peng Zhang, MD, ${ }^{\mathrm{f}}$ Chun Chen, MD, ${ }^{\mathrm{g}}$ Xinming Zhou, MD, ${ }^{\mathrm{h}}$ Yangchun Liu, MD, ${ }^{\mathrm{i}}$ \\ Keneng Chen, MD, Jianyong Ding, MD, ${ }^{\mathrm{j}}$ Yongtao Han, MD, ${ }^{\mathrm{l}}$ Yin Li, MD, ${ }^{\mathrm{m}}$ Zhentao Yu, MD, ${ }^{\mathrm{n}}$ \\ Yuan Liu, MS, ${ }^{a}$ Jianhua Fu, MD, ${ }^{\circ}$ and Members of the Chinese Alliance for Research in Thymomas*
}

\section{ABSTRACT}

Objectives: To study the incidence and pattern of lymph node metastases in thymic malignancies.

Methods: This multicenter prospective observational trial with intentional lymph node dissection was carried out by the Chinese Alliance for Research in Thymomas (ChART). Data on patients with thymic tumors without pretreatment were collected prospectively. Results from this prospective study were then compared with those from a previously reported ChART retrospective study.

Results: Among 275 patients, metastasis was detected in 41 nodes $(3.04 \%)$ in 15 patients $(5.5 \%)$. The rate of lymph node metastasis was $2.1 \%(5 / 238)$ in patients with thymomas, 25\% (6/24) in those with thymic carcinomas, and 50\% (4/8) in those with neuroendocrine tumors $(P<.001)$. The rate of lymph node metastasis in category T1 to T4 tumors was $2.7 \%(6 / 222)$ in $\mathrm{T} 1,7.7 \%(1 / 13)$ in $\mathrm{T} 2,18.4 \%(7 / 38)$ in $\mathrm{T} 3$, and $50 \%(1 / 2)$ in T4 $(P<.001)$. Nodal involvement was significantly higher compared with the ChART retrospective study $(5.5 \%$ vs $2.2 \% ; P=.002)$, although the 2 groups were comparable in terms of tumor stage and histology. Metastasis was found in N1 nodes in 13 patients $(86.7 \%)$ and in $\mathrm{N} 2$ nodes in 8 patients $(53.3 \%)$; 6 patients $(40 \%)$ had simultaneous N1/N2 diseases and $6(40 \%)$ had multistation involvement. Based on World Health Organization histological classification and Union for International Cancer Control T category, patients were divided into a low-risk group (1/192; $0.5 \%$ ) with T1-2 and type A-B2 diseases and a high-risk group $(14 / 83 ; 16.9 \%)$ of category T3 and above or histology B3 and above tumors for nodal metastasis $(P<.001)$. On multivariate analysis, type B3/thymic carcinoma/neuroendocrine tumors, category T3 or above, and N2 dissection predicted a greater likelihood of finding nodal metastasis.

Conclusions: Lymph node involvement in thymic malignancies is more common than previously recognized, especially in tumors with aggressive histology and advanced $\mathrm{T}$ category. Intentional lymph node dissection increases the detection of nodal involvement and improves accuracy of staging. In selected high-risk pa-

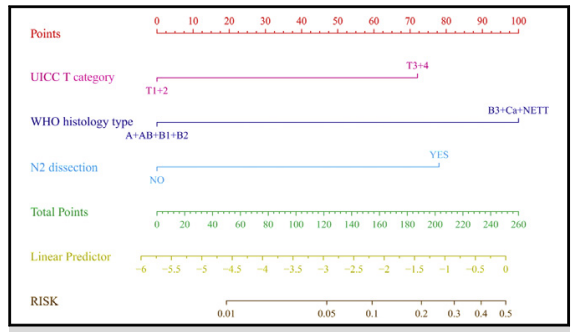

Nomogram predicting the detection of nodal metastasis in thymic tumors.

\section{Central Message}

Lymph node dissection should be considered in highrisk patients with high-grade tumors (World Health Organization type B3 thymoma, thymic carcinoma, and neuroendocrine thymic tumor) or local tumor extension (stage T3-4).

\section{Perspective}

Lymph node metastasis is a rare event in thymic tumors but is not uncommon in locally advanced or highergrade tumors. According to Union for International Cancer Control T category and World Health Organization histology, patients can be divided into a low-risk group $(0.5 \%)$ and a high-risk group $(16.9 \%)$ of nodal metastasis. Intentional lymph node dissection helps improve the accuracy of staging. Systemic nodal dissection should be considered in high-risk patients.

See Editorial Commentary page 834.

See Editorial page 822 . tients, systemic dissection of both N1and N2 nodes should be considered for accurate tumor staging. (J Thorac Cardiovasc Surg 2018;156:824-33)

From the ${ }^{a}$ Department of Thoracic Surgery, Shanghai Chest Hospital, Shanghai Jiao Tong University, Shanghai, China; 'b Department of Thoracic Surgery, West China Hospital, Sichuan University, Chengdu, China; ${ }^{\mathrm{c} D e p a r t m e n t}$ of Thoracic Surgery, Huashan Hospital, and ${ }^{\mathrm{k}}$ Department of Thoracic Surgery, Zhongshan Hospital, Fudan University, Shanghai, China; ${ }^{\mathrm{d}}$ Department of Thoracic Surgery, Affiliated Hospital of Qingdao University, Qingdao, China; ${ }^{\mathrm{D}}$ Department of Thoracic Surgery, Liaoning Cancer Hospital, Shenyang, China; ${ }^{\mathrm{f}}$ Department of Endocrinology, Tianjin Medical University General Hospital, Tianjin, China; ${ }^{g}$ Department of Thoracic Surgery, Fujian Medical University Union Hospital, Fuzhou, China; ${ }^{\mathrm{h}}$ Department of Thoracic Surgery, Zhejiang Cancer Hospital, Hangzhou, China; ${ }^{i}$ Department of Thoracic Surgery, Jiangxi People's Hospital, Nanchang, China; ${ }^{j}$ Department of Thoracic Surgery, Beijing Cancer Hospital, Beijing, China; ${ }^{1}$ Department of Thoracic Surgery, Sichuan Cancer Hospital, Chengdu, China; ${ }^{\mathrm{m}}$ Department of Thoracic Surgery, Affiliated Cancer Hospital of Zhengzhou University, Zhengzhou, China; ${ }^{\mathrm{n}}$ Department of Esophageal Cancer, Tianjin Cancer Hospital, Tianjin, China; and ${ }^{\circ}$ Department of Thoracic Surgery, Guangdong Esophageal Cancer Institute, Sun Yat-sen University Cancer Center, State Key Laboratory of Oncology in South China, Collaborative Innovation Center of Cancer Medicine, Guangzhou, China.

Drs Fang, Wang, and Pang contributed equally to this work.

Read at the 97th Annual Meeting of The American Association for Thoracic Surgery, Boston, Massachusetts, April 29-May 3, 2017

* A list of members of the Chinese Alliance for Research in Thymomas is provided in the Appendix E1.

Received for publication Aug 17, 2017; revisions received March 24, 2018; accepted for publication April 2, 2018; available ahead of print May 16, 2018

Address for reprints: Wentao Fang, MD, Department of Thoracic Surgery, Shanghai Chest Hospital, Shanghai Jiao Tong University, 241 Huaihai Rd West, Shanghai 200030, China (E-mail: vwtfang12@shchest.org). or Jianhua Fu, MD, Department of Thoracic Surgery, Sun Yat-sen University Cancer Center, 561 Dongfeng Rd East, Guangzhou, Guangdong 510060, China (E-mail: fujh@sysucc.org.cn). $0022-5223 / \$ 36.00$

Copyright (C) 2018 by The American Association for Thoracic Surgery https://doi.org/10.1016/j.jtcvs.2018.04.049 


\section{Abbreviations and Acronyms}

ChART $=$ Chinese Alliance for Research in Thymomas

CI = confidence interval

IASLC $=$ International Association for Studies in Lung Cancer

ITMIG = International Thymic Malignancy Interest Group

NETT $=$ neuroendocrine thymic tumor

OR = odds ratio

SEER = Surveillance, Epidemiology, and End Results

VATS $=$ video-assisted thoracic surgery

WHO $=$ World Health Organization

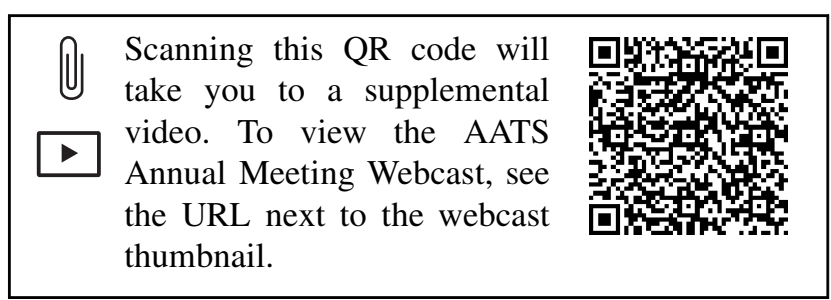

Thymic malignancies are relatively rare tumors compared with other malignant lesions of the chest. ${ }^{1}$ Because of their rarity, many clinicopathological features of these tumors still await to be elucidated, including the pattern of lymph node involvement. Only in recent years have there appeared some reports on the incidence of lymph node metastasis in thymic malignancy, with a great discrepancy in the results. ${ }^{2-7}$ The Chinese Alliance for Research in Thymomas (ChART) recently published a retrospective study based on its multicenter database of 1617 patients. ${ }^{8}$ The overall metastasis rate of a mere $2.2 \%$ seems to suggest that nodal involvement is rather uncommon in this relatively indolent tumor, especially in low-grade, early-stage thymic tumors. However, nodal involvement is not uncommon in tumors of a higher stage or higher-grade histology, especially in neuroendocrine thymic tumors (NETTs). In addition, nodal involvement seems to denote worse prognosis, in addition to tumor invasion and histology, indicating its significance in predicting prognosis and directing treatment.

Along with the scarcity of knowledge on lymph node spread, neither lymph node dissection nor systemic sampling has ever been recommended as a standard practice for thymic tumors. The International Association for Studies in Lung Cancer (IASLC)/International Thymic Malignancy Interest Group (IMTIG) proposal for the Union for International Cancer Control staging system proposed a new lymph node map for thymic malignancy. ${ }^{9}$ Lymph nodes located in the anterior mediastinum are defined as $\mathrm{N} 1$ nodes, and those in the deep mediastinum are defined as N2 nodes. If intentional lymphadenectomy were to be carried out in thymic tumors, there are no data indicating which subgroup of patients would be appropriate candidates or the extent to which lymph nodes should be harvested. Theoretically, in cases of total thymectomy, N1 nodes would have already been included in the resection area, but $\mathrm{N} 2$ nodes are usually left behind.

Based on these concerns, in the present study we aimed to further define the incidence and pattern of nodal metastases in thymic malignancies through intentional lymph node dissection/sampling.

\section{PATIENTS AND METHODS}

For this multicenter prospective observational study, Institutional Review Board approval was obtained from the participating hospitals, and written consent was acquired from all patients before surgery. Inclusion criteria were patients age from 15 to 80 years with an anterior mediastinal lesion clinically suspicious for thymic tumor and considered operable by the surgeon. Exclusion criteria were patients who had received any preoperative induction therapy for advanced tumors or those medically unfit for surgery. We deliberately excluded patients with preoperative therapies, because effective induction might downstage the tumor and lead to underestimation of the actual incidence of nodal metastasis. Patients who turned out to have a lesion other than thymic malignancy were then eliminated from the study.

All patients were operated on with an intention to cure. Thymectomy, instead of thymomectomy or tumor enucleation, was the standard procedure. In patients with myasthenia gravis, extended thymectomy was performed. The surgical approach (open or minimally invasive, median or lateral incision) was chosen according to the surgeon's preference without any special requirement for lymph node dissection, except that transcervical incision was considered unacceptable for nodal retrieval. The minimal request for this study was that at least 1 anterior mediastinal lymph node station close to the primary lesion be intentionally harvested and sent for histological examination (Video 1). However, there was no definite requirement as to the number or extent (unilateral or bilateral, $\mathrm{N} 1$ or $\mathrm{N} 2$, according to the IASLC/ITMIG definition) of lymph node dissection

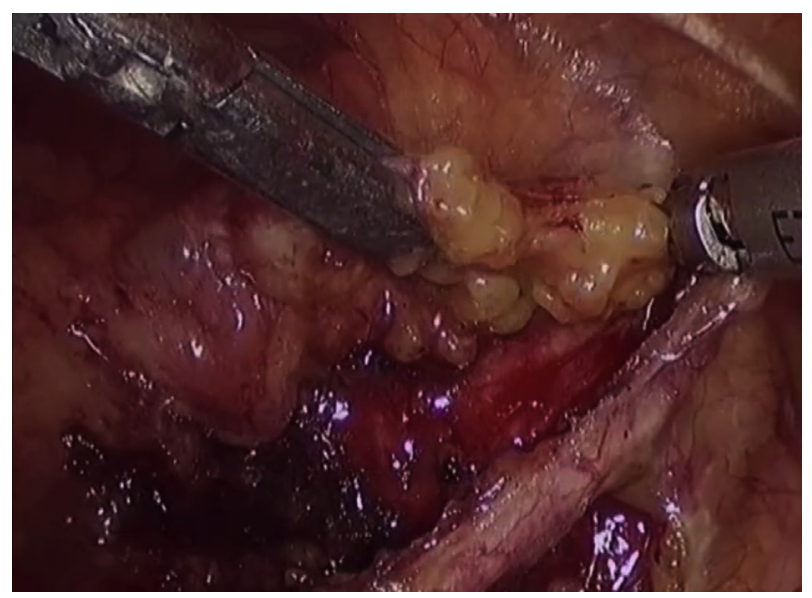

VIDEO 1. Lymph node sampling via a bilateral video-assisted thoracic surgery approach. Video available at: https://www.jtcvs.org/article/ S0022-5223(18)31034-1/fulltext. 
or sampling, although surgeons were encouraged to harvest as many nodes as possible.

Laterality, local extension, and histology subtype of the primary tumor were documented to study the relationships between different tumors and patterns of lymph node involvement. Tumors with their epicenter lateral to the internal mammary vessels on one side on preoperative computed tomography scan were considered laterally located, whereas tumors with their epicenter between the bilateral internal mammary vessels were considered centrally located. Tumor staging was based on the IASLC/ITMIG proposal for the Union for International Cancer Control staging system. ${ }^{10}$ Tumor histology was classified according to World Health Organization (WHO) type ${ }^{11}$ with NETT considered an individual subgroup. Results from this prospective study were then compared with those from a previously reported ChART retrospective database study. ${ }^{8}$

Statistical analysis was performed using SPSS 17.0 (SPSS, Chicago, III). Categorical variables were analyzed using the $\chi^{2}$ test, or the Fisher exact test for small samples, for differences between the groups. Continuous data were compared using the Student $t$ test. To identify potential risk factors predicting nodal involvement, univariate binary logistic regression was performed, followed by a multivariable analysis including only variables with $P<.10$ in the univariate analysis. Statistical significance was accepted as $P<.05$ throughout the study.

\section{Nomogram Construction}

We generated a predictive nomogram model to predict high-risk patients. Then, based on the results of the multivariable analysis, variables that achieved significance at $P<.05$ were selected to formulate a nomogram using R 3.4.1 (http://www.r-project.org) with the rms package.

\section{RESULTS}

A total of 275 patients with thymic tumors who underwent surgical resection at 15 tertiary referral centers in the ChART group between June 1, 2014, and August 31, 2016, were entered into the study. The cohort included 130 males $(47.3 \%)$ and 145 females $(52.7 \%)$, with a mean age of 53.9 years (range, 18-80 years). Fifty-six patients $(20.4 \%)$ had a concomitant autoimmune disease, the vast majority with myasthenia gravis $(\mathrm{n}=52)$. The patients had a total of 243 thymomas $(88.4 \%), 24$ thymic carcinomas $(8.7 \%)$, and 8 NETTs $(2.9 \%)$. Surgical approaches included 134 open procedures $(48.4 \%)$ and 141 video-assisted thoracic surgery (VATS) resections $(51.3 \%)$. Bilateral lymph nodal dissection/sampling was carried out in $11.8 \%(16 / 135)$ of patients undergoing open surgery and in 5\% (7/141) of those undergoing a VATS procedure. No surgical mortality or major complications directly related to lymph node harvesting were recorded during the study. Complete resection was confirmed by postoperative histological examination in 268 cases $(97.5 \%)$. Pathological T1a, $\mathrm{T} 1 \mathrm{~b}, \mathrm{~T} 2, \mathrm{~T} 3$, and $\mathrm{T} 4$ tumors were identified in 198 $(72 \%), 24(8.7 \%), 13(4.7 \%), 38(13.8 \%)$, and 2 $(0.7 \%)$ patients, respectively.

A total of 1347 lymph nodes from 847 stations were harvested, with a mean number of 4.9 nodes and 3.1 stations per patient (Figure 1). Patients undergoing VATS surgery actually had a greater number of lymph nodes (5.31 vs $4.46 ; P=.05)$ and more stations (3.30 vs $2.84 ; P=.017)$ harvested, and more $\mathrm{N} 2$ dissections (1.57 vs 1.45 ; $P=.048$ ) compared with patients undergoing open procedures. On histological examination, nodal metastasis was detected in 41 nodes $(3.04 \%)$ in 15 patients $(5.5 \%)$. Demographic characteristics, surgical approaches, and extent of nodal retrieval in patients with or without nodal disease are presented in Table 1. Overall, the nodal metastasis rate was significantly higher than the $2.2 \%$ rate $(35 /$ $1617 ; P=.002)$ reported in the retrospective ChART study, ${ }^{8}$ although no significant difference in the percentage of histological subtypes $(P=.102)$ or $\mathrm{T}$ categories $(P=.188)$ was detected between the retrospective and prospective databases (data not shown). Compared with the results of the retrospective study, nodal involvement was much more common in most histology types and $\mathrm{T}$ categories in stratified analysis (Table 2). The overall metastasis rate was $2.1 \%$, in thymomas, $25 \%$ in thymic carcinomas, and $50 \%$ in NETTs $(P<.05)$, and $2.0 \%$ in T1a tumors, $8.3 \%$ in T1b tumors, $7.7 \%$ in T2 tumors, $18.4 \%$ in T3 tumors, and $50 \%$ in $\mathrm{T} 4$ tumors $(P<.001)$. No lymph node involvement was found in type A or B1 thymomas. Stations of lymph nodes harvested and lymph node dissection rates are listed in Table 3. No significant difference in lymph node dissection rate was detected between $\mathrm{N} 1$ and $\mathrm{N} 2$ nodes (3.1\% vs $2.7 \% ; P=.743)$.

In univariate analysis, WHO histology, T category, tumor size, and bilateral and $\mathrm{N} 2$ node sampling were significantly associated with positive nodal disease. In multivariate analysis, WHO type B3/thymic carcinoma/NETT $(P=.001$; odds ratio [OR], 9.857; 95\% confidence interval [CI], 2.522-38.521), category T3-4 ( $P=.009$; OR, 2.281; $95 \%$ CI, 1.229-4.23), and N2 node dissection $(P=.015$; OR, 5.96; $95 \% \mathrm{CI}, 1.418-25.05)$ independently predicted a greater likelihood of finding nodal metastases (Table 4). Based on these 3 factors, a nomogram predicting nodal involvement was built (Figure 2), which calculates the risk of nodal disease as follows:

$$
\mathrm{LN}(\text { Risk })=-5.738+1.649 \mathrm{~T}+2.288 \mathrm{WHO}+1.785 \mathrm{~N} 1 / 2 .
$$

According to histology and $\mathrm{T}$ category alone, patients could be divided into a low-risk group, with stage T1-2 and type $\mathrm{A} / \mathrm{AB} / \mathrm{B} 1 / \mathrm{B} 2$ thymomas, and a high-risk group, with stage $\mathrm{T} 3$ and above or higher-grade histology (B3 thymoma, thymic carcinoma, NETT). N2 node dissection was carried out in $44.3 \%(85 / 192)$ of patients in the low-risk group and in $36.1 \%$ (30/83) of patients in the high-risk group $(P=.210)$. The low-risk group accounted for $69.8 \%(192 / 275)$ of the study population, with lymph node involvement seen in only 1 patient $(0.5 \%)$ with a giant type $\mathrm{AB}$ tumor. In contrast, nodal metastasis was $16.9 \%$ (14/83) in the high-risk group, which 

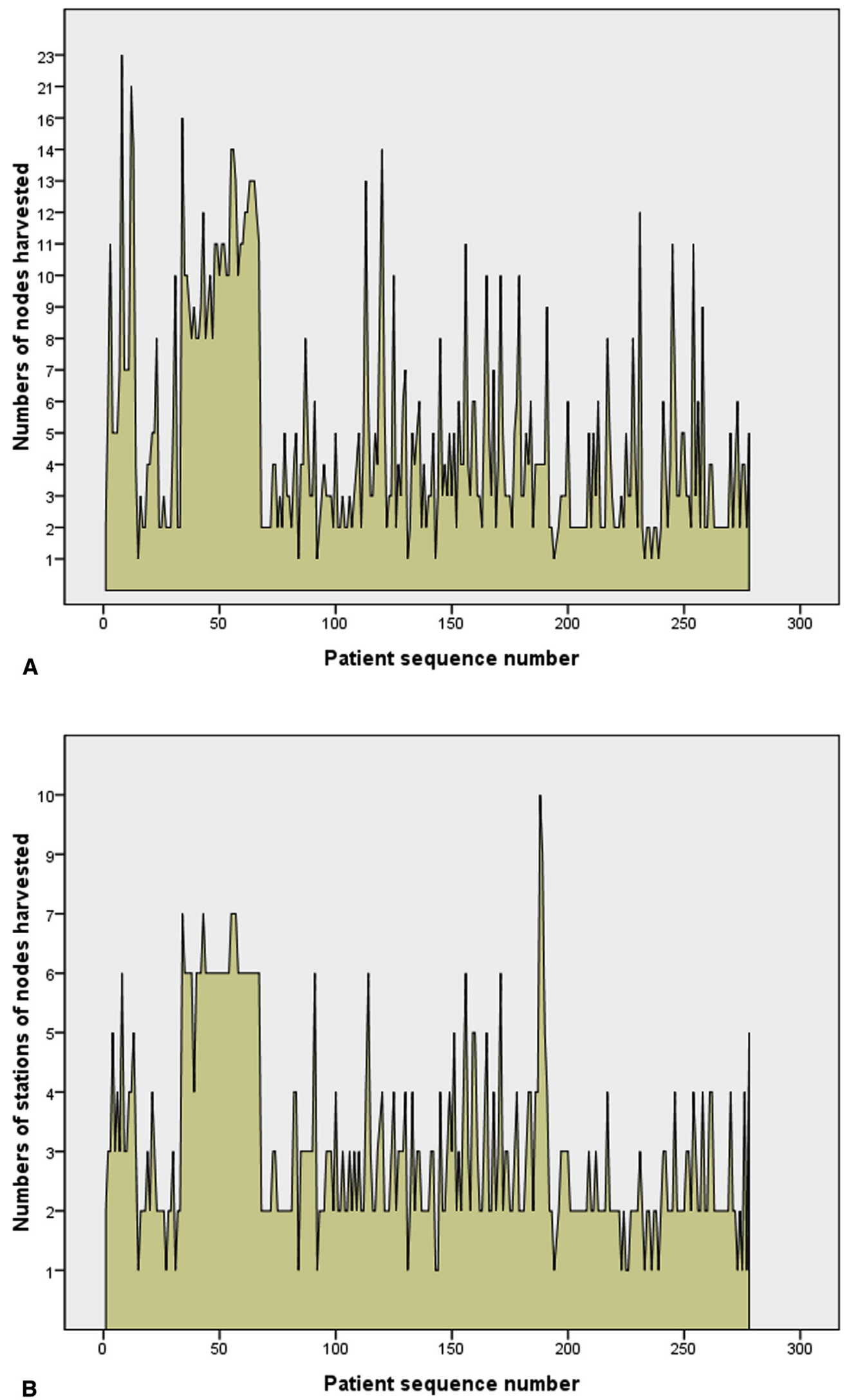

FIGURE 1. Numbers of nodes and stations of nodes harvested per patient. A, Numbers of nodes harvested per patient. B, Numbers of stations of nodes harvested per patient. 
TABLE 1. Demographic characteristics of the patients, surgical approaches, and extent of tumor resection

\begin{tabular}{|c|c|c|c|}
\hline Characteristic & $\begin{array}{c}\mathbf{N}(-) \\
(\mathbf{n}=\mathbf{2 6 0})\end{array}$ & $\begin{array}{c}\mathbf{N}(+) \\
(\mathbf{n}=\mathbf{1 5})\end{array}$ & $P$ value \\
\hline Age, $y$, mean \pm SD & $53.85 \pm 11.8$ & $53.87 \pm 9.9$ & .349 \\
\hline \multicolumn{4}{|l|}{ Sex, n $(\%)$} \\
\hline Male & $124(95.4)$ & $6(4.6)$ & .562 \\
\hline Female & $136(93.8)$ & $9(6.2)$ & \\
\hline Tumor size, $\mathrm{cm}$, mean $\pm \mathrm{SD}$ & $5.57 \pm 2.65$ & $7.5 \pm 4.03$ & .005 \\
\hline $\begin{array}{l}\text { Preoperative autoimmune } \\
\text { disease, } \mathrm{n}(\%)\end{array}$ & $54(96.4)$ & $2(3.6)$ & .743 \\
\hline \multicolumn{4}{|l|}{ Surgical approach, n (\%) } \\
\hline Open & $129(91.8)$ & $11(8.2)$ & .063 \\
\hline VATS & $137(97.2)$ & $4(2.8)$ & \\
\hline $\mathrm{T}$ category, $\mathrm{n}(\%)$ & & & $<.001$ \\
\hline T1a & $194(98)$ & $4(2.0)$ & \\
\hline T1b & $22(91.7)$ & $2(8.3)$ & \\
\hline $\mathrm{T} 2$ & $12(92.3)$ & $1(7.7)$ & \\
\hline T3 & $31(83.8)$ & $7(18.4)$ & \\
\hline $\mathrm{T} 4$ & $1(50)$ & $1(50)$ & \\
\hline WHO histology type, n (\%) & & & $<.001$ \\
\hline A-B2 & 204 (98.6) & $3(1.4)$ & \\
\hline B3 & $34(94.4)$ & $2(5.6)$ & \\
\hline Carcinoma & $18(75)$ & $6(25)$ & \\
\hline NETT & $4(50)$ & $4(50)$ & \\
\hline \multicolumn{4}{|l|}{ LN dissection, $\mathrm{n}(\%)$} \\
\hline Unilateral & $158(97.5)$ & $4(2.5)$ & .013 \\
\hline Bilateral & $102(90.3)$ & $11(9.7)$ & \\
\hline N1 only & $131(97.8)$ & $3(2.2)$ & .022 \\
\hline $\mathrm{N} 1+\mathrm{N} 2$ & $129(91.5)$ & $12(8.5)$ & \\
\hline
\end{tabular}

$N(-)$, Without nodal involvement; $N(+)$, with nodal metastasis; $S D$, standard deviation; VATS, video-assisted thoracic surgery; $W H O$, World Health Organization; $N E T T$, neuroendocrine thymic tumor; $L N$, lymph node.

accounted for only $30.2 \%$ (83/275) of all patients enrolled in this study, significantly more common than in the lowrisk group $(P<.001)$.

Among the 15 patients with nodal diseases, metastasis was found in $\mathrm{N} 1$ nodes in 13 patients $(86.7 \%)$ and in N2 nodes in 8 patients $(53.3 \%)$. Six of these 15 patients $(40 \%)$ had simultaneous N1/N2 disease, and $6(40 \%)$ had multistation involvement (Table 5). Broken down by tumor stage and histology, only 1 of 207 patients $(0.5 \%)$ with a type A-B2 tumor had N2 involvement, compared with 3 of 43 patients $(6.9 \%)$ with a T1-2 tumor and 4 of $25(16 \%)$ with a T3-4 type B3/C/NETT tumor. Three of the 15 patients $(20 \%)$ had bilateral nodal metastasis, all with NETTs (atypical carcinoid), and all had simultaneous N1/N2 diseases. Of the 5 patients with unilateral $\mathrm{N} 2$ involvement, 4 had a laterally located tumor, and all 4 had node involvement on the ipsilateral side of the primary tumor. In 1 patient with unilateral $\mathrm{N} 2$ involvement, the tumor was a centrally located squamous cell carcinoma invading into bilateral lung. When N2 node dissection was planned, the risk of
TABLE 2. Comparison of lymph node metastasis rates in different histology types and $\mathrm{T}$ categories between the ChART retrospective and prospective studies

\begin{tabular}{lccc}
\hline & \multicolumn{2}{c}{ Nodal metastasis, $\mathbf{n} / \mathbf{N}(\%)$} & \\
\cline { 2 - 3 } Variable & $\begin{array}{c}\text { ChART } \\
\text { retrospective }\end{array}$ & $\begin{array}{c}\text { ChART } \\
\text { prospective }\end{array}$ & P value \\
\hline WHO histology & & & \\
A & $0 / 98(0)$ & $0 / 21(0)$ & - \\
AB & $0 / 424(0)$ & $2 / 83(2.4)$ & .025 \\
Micronodular & $0 / 2(0)$ & $0 / 6(0)$ & - \\
B1 & $0 / 245(0)$ & $0 / 36(0)$ & - \\
B2 & $1 / 275(0.4)$ & $1 / 61(1.6)$ & .358 \\
B3 & $6 / 266(2.3)$ & $2 / 36(5.6)$ & .244 \\
Ca & $21 / 265(7.9)$ & $6 / 24(25)$ & .006 \\
NETT & $7 / 42(16.7)$ & $4 / 8(50)$ & .037 \\
T category & & & \\
T1a & $3 / 1124(0.3)$ & $4 / 198(2.0)$ & .002 \\
T1b & $0 / 99(0)$ & $2 / 24(8.3)$ & .037 \\
T2 & $5 / 72(6.9)$ & $1 / 13(7.7)$ & .923 \\
T3 & $25 / 295(8.5)$ & $7 / 38(18.4)$ & .050 \\
T4 & $2 / 27(7.4)$ & $1 / 2(50)$ & .056 \\
\hline
\end{tabular}

ChART, Chinese Alliance for Research in Thymomas; WHO, World Health Organization; $\mathrm{Ca}$, thymic carcinoma; $N E T T$, neuroendocrine thymic tumor.

revealing $\mathrm{N} 2$ nodal metastasis according to the nomogram was 0.019 for WHO type A-B2 and category T1-2 tumors, 0.099 for type A-B2 and T3-4 tumors, 0.189 for type B3NETT and T1-2 tumors, and 0.984 for type B3-NETT and T3-4 tumors.

\section{DISCUSSION}

In this multicenter prospective observational study with intentional lymph node retrieval during total thymectomy, we detected a significantly higher incidence of lymph node involvement than in the previously reported ChART retrospective study. ${ }^{8} \mathrm{~N} 2$ node dissection, along with higher-grade tumor histology and advanced $\mathrm{T}$ category, were found to be associated with increased nodal-positive rate. Patients with thymic tumors could be divided into high-risk and low-risk subgroups depending on tumor histology and $\mathrm{T}$ category, suggesting that selective dissection may be warranted.

Lymph node dissection for malignant tumors is performed to increase the accuracy of tumor staging, to ensure radical removal of the disease, and to improve surgical outcomes. However, intentional lymph node dissection or sampling is seldom recommended as a routine procedure for thymic malignancies. In this study, the overall rate of lymph node involvement of $5.5 \%(15 / 275)$ is more than double the $2.2 \%$ rate $(35 / 1617 ; P=.002)$ in a previously reported retrospective analysis of the same study group without lymph node dissection. ${ }^{8}$ Although there was no significant difference in histological subtype or T category distribution between the retrospective and prospective databases, we 
TABLE 3. Stations of lymph nodes dissected/sampled and rate of nodal involvement at each station

\begin{tabular}{lcccc}
\hline \multicolumn{1}{c}{ Node station } & IASLC level & ITMIG map & LNs harvested, $\mathbf{n}(\%)$ & LN dissection rate, $\mathbf{n}(\%) *$ \\
\hline Prevascular & $3 \mathrm{a}$ & $\mathrm{N} 1$ & $239(86.9)$ & $5(2.1)$ \\
Upper paratracheal & 2 & $\mathrm{~N} 2$ & $94(34.2)$ & $3(3.7)$ \\
\hline Lower paratracheal & 4 & $\mathrm{~N} 2$ & $94(34.2)$ & $1(1.1)$ \\
Right hilum & $\mathrm{R} 10$ & $\mathrm{~N} 2$ & $50(18.2)$ & $1(2)$ \\
Para-aortic & 6 & $\mathrm{~N} 1$ & $219(79.6)$ & $9(4.1)$ \\
Subaortic & 5 & $\mathrm{~N} 2$ & $100(36.3)$ & $4(4)$ \\
Left hilum & L10 & N2 & $36(13.1)$ & $1(2.8)$ \\
\hline
\end{tabular}

IASLC, International Association for Studies in Lung Cancer; ITMIG, International Thymic Malignancy Interest Group; $L N$, lymph node. *Lymph node dissection rate at each station $=$ number of patients with nodal metastasis/number of patients with nodes harvested at that station

compared the rates of nodal metastasis in each WHO histology type and $\mathrm{T}$ category between the 2 cohorts to further exclude potential selection biases. No lymph node involvement was found in type A or B1 tumors in either study. Compared with the ChART retrospective study, the rate of nodal metastasis was much higher for all other histology types and each $\mathrm{T}$ category in the prospective study. The differences between the 2 cohorts were statistically significant except for type B2 and B3 and category T2 tumors, likely because of the small number of total and node-positive cases. This clearly indicates that intentional lymph node retrieval would increase the yield of detection of nodal diseases in thymic tumors, and thus is at least important for accurate staging and radical removal of the disease.

In the present study, lymph node metastasis was seen in $2.1 \%$ of patients with thymomas, in $25 \%$ of those with thymic carcinomas, and in $50 \%$ of those with NETTs. The highest rates of lymph node involvement reported to date were from 2 retrospective studies using the Surveillance, Epidemiology, and End Results (SEER) database, 5,6 although accurate comparisons among different studies are difficult. In a study including only patients with at least 1 lymph node harvested for histological examination, Weksler and colleagues ${ }^{5}$ reported rates of lymph node spread of $33.5 \%$ in thymic carcinomas and $62.3 \%$ in NETTs, similar to our results. In another study reported by the same authors, the rate of nodal metastasis was surprisingly high in thymomas $(13.3 \%) .{ }^{6}$ However, more than one-half of the tumors in their cohort were Masaoka stage $>$ III, whereas $>85 \%$ of the tumors in our series were in category T1-2, which corresponds to Masaoka stage I-II. This discrepancy is understandable, because the SEER database is basically a cancer registry. Moreover, our understanding of thymic malignancy has changed significantly, and all thymic tumors are now considered malignant in nature. ${ }^{12}$ Thus, cases recorded in the SEER database might not represent the general picture of these tumors. In fact, the higher rate of nodal involvement in the series of Weksler and colleagues also supports our finding that increased nodal involvement is associated not only with tumor histology, but also with the extent of local invasion of the primary lesion. Both

TABLE 4. Univariate and multivariate analysis for potential risk factors predicting lymph node involvement in thymic tumors

\begin{tabular}{lrr}
\hline \multicolumn{1}{c}{ Variable } & OR $(\mathbf{9 5} \% \mathbf{C I})$ & $\boldsymbol{P}$ value \\
\hline Univariate analysis & & .997 \\
Age & $1.000(0.956-1.046)$ & .563 \\
Sex, male vs female & $0.731(0.253-2.113)$ & .011 \\
Tumor size & $1.236(1.049-1.455)$ & .492 \\
Preoperative autoimmune disease, no vs yes & $1.704(0.373-7.779)$ & $<.001$ \\
WHO histology, B3-C vs A-B2 & $14.571(3.974-53.427)$ & $<.001$ \\
T category, T3-4 vs T1-2 & $8.143(2.766-23.972)$ & .057 \\
Surgical approach, open vs VATS & $3.111(0.965-10.023)$ & .015 \\
LN dissection (bilateral vs unilateral) & $4.26(1.321-13.741)$ & .033 \\
LN dissection (N1 + N2 vs N1 only) & $4.062(1.12-14.731)$ & .001 \\
Multivariate analysis & & .009 \\
WHO histology type (B3-C vs A-B2) & $9.857(2.522-38.521)$ & .015 \\
T category (T3-4 vs T1-2) & $2.281(1.229-4.23)$ & \\
LN dissection (N1+ N2 vs N1 only) & $5.96(1.418-25.05)$ & \\
\hline
\end{tabular}

$O R$, Odds ratio; $C I$, confidence interval; $W H O$, World Health Organization; $L N$, lymph node. 


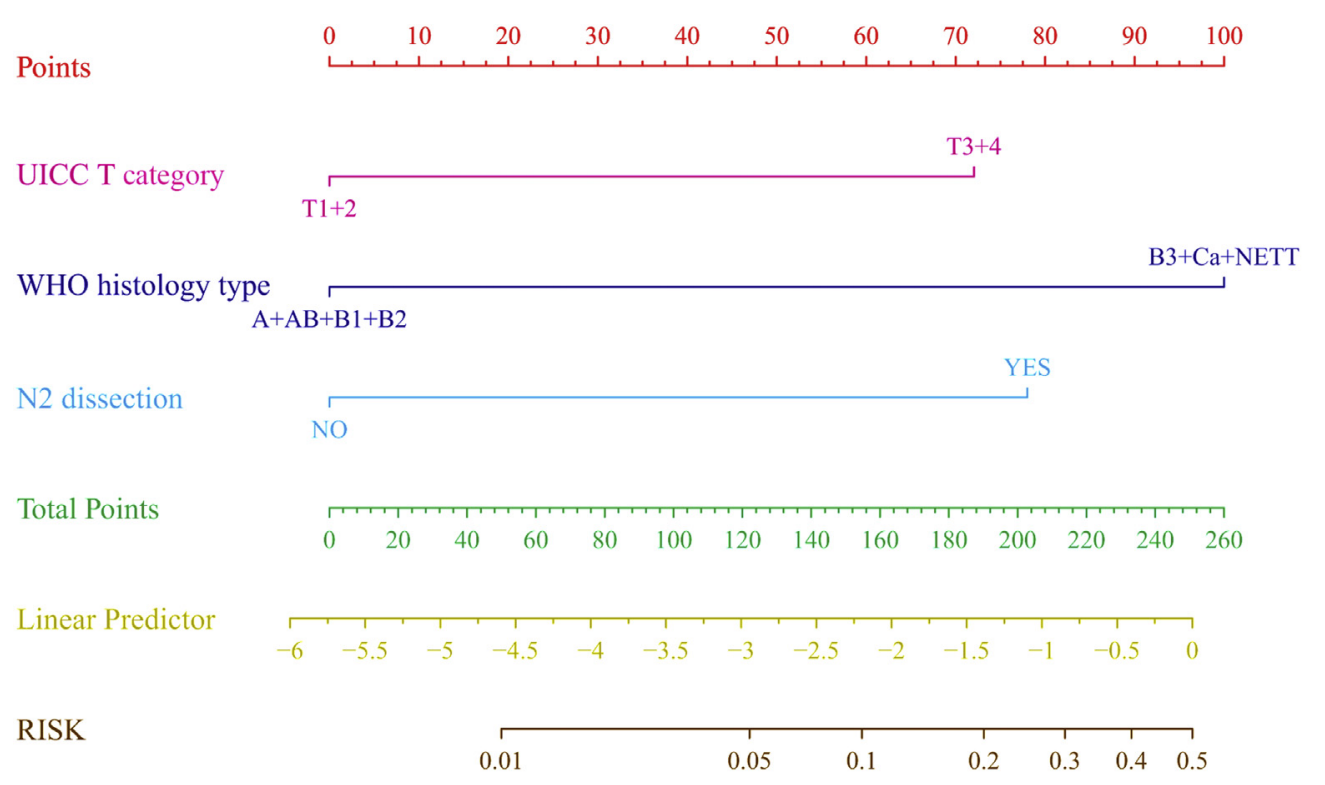

FIGURE 2. A nomogram predicting detection of positive nodal status in thymic tumor patients. UICC, Union for International Cancer Control; WHO, World Health Organization; $\mathrm{Ca}$, thymic carcinomas; NETT, neuroendocrine thymic tumors.

factors should be considered when predicting the likelihood of lymphatic spread in these patients.

According to our results, the risk of lymph node metastasis was not associated with patient age or sex or the presence of autoimmune disease. Tumor size differed between patients with nodal disease and those without nodal disease in univariate analysis but not in multivariate analysis. Apart from N2 dissection, only tumor histology and $\mathrm{T}$ category were independent predictors for nodal involvement. This finding is in accordance with our previous study using the ChART retrospective database. ${ }^{8}$ Based on these results, patients could be divided into a low-risk group with nodal metastasis and a high-risk group with tumors with local invasion into the neighboring organs (stage $\mathrm{T} 3$ and above) or with higher-grade histology (B3 thymoma, thymic carcinoma, or NETT). Only 1 patient $(0.5 \%)$ in the low-risk group $(69.8 \%$ of the study population) had nodal disease, whereas $16.9 \%$ of the high-risk patients $(30.2 \%$ of the study population) already had lymph node metastasis. If intentional lymphadenectomy were carried out selectively only in the high-risk group, nearly $70 \%$ of the patients with thymic tumors could be exempted from unnecessary dissection. It is also noteworthy that the frequency of N2 node dissection was similar in these 2 groups; therefore, it is unlikely that the extent of dissection accounted for the different rates of nodal involvement in these patients.

Another important point when considering lymph node evaluation is the extent of nodal dissection. Among different regions there seems to be a preference of surgical approaches for thymic tumors (VATS vs open; median vs unilateral). ${ }^{13}$ Whether this would have any impact on lymph node retrieval is unknown. In this study, we did not find any significant association between surgical approach and lymph node yield. This result is highly relevant to the choice of surgical procedure, with minimally invasive surgery increasingly used for thymic tumors, ${ }^{14}$ with a unilateral approach in the majority of these procedures. Although most tumors selected for VATS or robot-assisted surgery are early-stage lesions that would fall into our low-risk group, the rate of nodal involvement was still $2.7 \%$ in $\mathrm{T} 1$ tumors and $7.7 \%$ in $\mathrm{T} 2$ tumors. It is noteworthy that all the patients in this study underwent total thymectomy, including those who underwent tumors resection by VATS. Theoretically, N1 nodes, located in the anterior mediastinum, would have already been removed before the entire thymus was resected via total thymectomy.

$\mathrm{N} 2$ node harvesting is even less often included in surgical resection of thymic tumors, unless performed intentionally. Whether it is indeed necessary is never known. In this study, the rates of $\mathrm{N} 1(4.7 \%)$ and $\mathrm{N} 2(2.9 \%)$ metastasis seemed to be similar, and $40 \%$ of the patients with nodal involvement had simultaneous N1/N2 disease. In addition, we found that the lymph node dissection rate, defined as the number of patients with nodal metastasis divided by the number of patients with that node station harvested, was similar between the $\mathrm{N} 1$ region $(3.1 \%)$ and $\mathrm{N} 2$ region $(2.7 \%$; $P=.743)$. Moreover, $\mathrm{N} 2$ dissection turned out to be another independent predictive factor for nodal disease. This would suggest that once lymph node metastasis occurs in thymic tumors, it tends to be multistational and often involves both $\mathrm{N} 1$ and $\mathrm{N} 2$ nodes. If lymph node dissection is deemed necessary, both $\mathrm{N} 1$ and $\mathrm{N} 2$ nodes should be harvested to 
TABLE 5. Clinical characteristics of 15 patients with lymph node metastasis

\begin{tabular}{|c|c|c|c|c|c|c|c|}
\hline Patient & WHO histology & Clinical stage & Surgical approach & Tumor size, cm & $\begin{array}{c}\text { Pathological } \\
\text { stage }\end{array}$ & $\begin{array}{c}\text { Extent of } \\
\text { lymphadenectomy }\end{array}$ & $\begin{array}{c}\text { Location of } \\
\text { positive LNs } \\
\text { (IASLC level) }\end{array}$ \\
\hline 1 & $\mathrm{AB}$ & T3NOMO & Open & $9 \times 9 \times 5$ & T3N1M0 & $\mathrm{N} 2$ & L6 \\
\hline 2 & $\mathrm{AB}$ & T3NOM0 & Open & $15 \times 11 \times 8$ & T1aN2M0 & N2 & $\mathrm{R} 10$ \\
\hline 3 & B2 & T3N0M1a & Open & $8.5 \times 5 \times 3$ & T3N1M1a & N1 & L6 \\
\hline 4 & B3 & T3NOM0 & Open & $4 \times 5 \times 4$ & T4N2M1a & $\mathrm{N} 2$ & L6, L5 \\
\hline 5 & B3 & T1N0M0 & VATS & $4 \times 3 \times 2.5$ & T1aN2M0 & $\mathrm{N} 2$ & L5 \\
\hline 6 & $\mathrm{Ca}$ & T1bN0M0 & Open & $7 \times 5 \times 4$ & T3N1M0 & $\mathrm{N} 2$ & L6 \\
\hline 7 & $\mathrm{Ca}$ & T1aN0M0 & VATS & $4 \times 2 \times 3$ & T1aN2M0 & $\mathrm{N} 2$ & L6, L5 \\
\hline 8 & $\mathrm{Ca}$ & T3NOM0 & Open & $7 \times 4.5 \times 4$ & T3N1M0 & N1 & R3a \\
\hline 9 & $\mathrm{Ca}$ & T3N0M1a & Open & $4 \times 3 \times 2$ & $\mathrm{~T} 2 \mathrm{~N} 1 \mathrm{M} 1 \mathrm{a}$ & N2 & L6 \\
\hline 10 & $\mathrm{Ca}$ & T3NOM0 & Open & $5 \times 4.5 \times 3.6$ & T3N1M1a & N1 & R3a \\
\hline 11 & $\mathrm{Ca}$ & T4N2M0 & Open & $13 \times 10 \times 5$ & T3N2M0 & $\mathrm{N} 2$ & L6, L5 \\
\hline 12 & NETT & T2NOM0 & VATS & $4.5 \times 3.5 \times 3$ & T1bN1M0 & N2 & $\mathrm{R} 3 \mathrm{a}$ \\
\hline 13 & NETT & T3NOM0 & Open & $9.5 \times 8 \times 4$ & T3N2M0 & N2 & R2, R3a, L5 \\
\hline 14 & NETT & T1N0M0 & Open & $15 \times 8.5 \times 8$ & T1aN2M0 & $\mathrm{N} 2$ & L6, L10, R2 \\
\hline 15 & NETT & T1bN0M0 & Open & $5 \times 4 \times 4$ & $\mathrm{~T} 1 \mathrm{bN} 2 \mathrm{M} 0$ & $\mathrm{~N} 2$ & R2, R3a, R4, L6 \\
\hline
\end{tabular}

WHO, World Health Organization; $L N$, lymph node; IASLC, International Association for Studies in Lung Cancer; VATS, video-assisted thoracic surgery; $C a$, thymic carcinoma; $N E T T$, neuroendocrine thymic tumor.

ensure complete resection and accurate staging. This should apply only to a selected group of high-risk patients, however. According to the nomogram that we built to predict nodal involvement, the risk of revealing $\mathrm{N} 2$ nodal metastasis would be only 0.019 for WHO type A-B2 and category T1-2 tumors, but 0.984 for type B3-NETT and T3-4 tumors.

Our results also showed that $\mathrm{N} 2$ involvement was usually on the ipsilateral side of tumor extension, except for NETTs. This seems to suggest that for thymomas and thymic carcinomas, only $\mathrm{N} 2$ nodes on the side of tumor invasion need to be retrieved. According to our results, at least stations 2 and 4 on the right side or station 5 on the left side, depending on the laterality of the primary lesion, should be harvested if $\mathrm{N} 2$ dissection is considered necessary. Hilar nodes on the ipsilateral side of the tumor should be included as well. The fact that bilateral dissection did not turn out to be an independent risk factor in multivariate analysis for nodal disease also supports this idea. In fact, all 3 patients with bilateral nodal involvement had NETT (atypical thymic carcinoid) and simultaneous N1/N2 metastasis, and 2 of them had bilateral N2 disease. Given that in both the prospective and retrospective studies, patients with NETT had the highest rate of lymph node involvement, special attention should be given to more extensive lymph node dissection in this group of patients. Because biopsy is usually not recommended for upfront surgery in patients with thymic malignancy, intraoperative frozen sections may be used to determine tumor histology and the extent of nodal dissection onsite.
One limitation of this study is the relatively small number of patients with thymic carcinoma and NETT. Nonetheless, nodal metastasis was found to be significantly higher in these subtypes, and both thymic carcinoma and NETT were identified as important risk factors for predicting nodal status. In addition, our study did not specify the extent of lymph node harvesting during thymectomy, and one-half of the patients did not have $\mathrm{N} 2$ nodal dissection. This would certainly limit our ability to more precisely define the pattern of lymph node involvement. Moreover, the small number of cases with nodal metastasis also might affect the accuracy of risk factor analysis. However, this is the sole prospective study with intentional lymph node retrieval in thymic tumors. In contrast to all other retrospective studies on the same topic, we managed to accrue a large patient cohort in a very limited study period. This also helped put the risk of confounding bias caused by evolution in surgical techniques to a minimum.

In conclusion, we found a significantly increased rate of lymph node metastasis in thymic tumors after intentional lymph node dissection/sampling. Once nodal metastasis occurred, it was often multistational, involving both $\mathrm{N} 1$ and N2. Our results suggest that lymph node dissection should be recommended in high-risk patients with high-grade tumors (WHO type B3 thymoma, thymic carcinoma, and NETT) or local extension (category T3-4). In addition to total thymectomy for N1 nodes, $\mathrm{N} 2$ nodes, especially those on the ipsilateral side of tumor extension, should be harvested as well. Bilateral 
dissection is usually unnecessary, except for the rare cases of neuroendocrine tumors with a high prevalence of extensive nodal involvement. These factors should be taken into consideration when designing surgical procedures for thymic malignancy.

\section{Webcast}

You can watch a Webcast of this AATS meeting presentation by going to: https://aats.blob.core.windows.net/ media/17AM/2017-05-03/RM312/05-03-17_Room312_0926_ Fang.mp4.

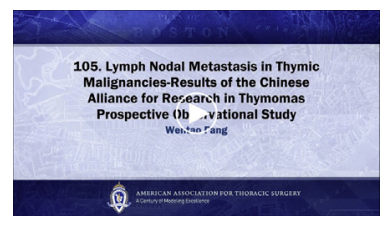

\section{Conflict of Interest Statement}

Authors have nothing to disclose with regard to commercial support.

We thank Clifford Harbutt for the language revision.

\section{References}

1. Engels EA. Epidemiology of thymoma and associated malignancies. J Thorac Oncol. 2010;5(10 Suppl 4):S260-5.

2. Kondo K, Monden Y. Lymphogenous and hematogenous metastasis of thymic epithelial tumors. Ann Thorac Surg. 2003;76:1859-64; discussion 1864-5.

3. Park IK, Kim YT, Jeon JH, Kim HS, Hwang Y, Seong YW, et al. Importance of lymph node dissection in thymic carcinoma. Ann Thorac Surg. 2013;96:1025-32; discussion 1032

4. Viti A, Bertolaccini L, Terzi A. What is the role of lymph nodal metastases and lymphadenectomy in the surgical treatment and prognosis of thymic carcinomas and carcinoids? Interact Cardiovasc Thorac Surg. 2014;19:1054-8.

5. Weksler B, Holden A, Sullivan JL. Impact of positive nodal metastases in patients with thymic carcinoma and thymic neuroendocrine tumors. J Thorac Oncol. 2015;10:1642-7.

6. Weksler B, Pennathur A, Sullivan JL, Nason KS. Resection of thymoma should include nodal sampling. J Thorac Cardiovasc Surg. 2015;149:737-42.

7. Hwang Y, Park IK, Park S, Kim ER, Kang CH, Kim YT. Lymph node dissection in thymic malignancies: implication of the ITMIG lymph node map, TNM stage classification, and recommendations. $J$ Thorac Oncol. 2016;1:108-14.

8. Gu Z, Wei Y, Fu J, Tan L, Zhang P, Han Y, et al. Lymph node metastases in thymic malignancies: a Chinese alliance for research in thymomas retrospective database analysis. Interact Cardiovasc Thorac Surg. 2017;25: 455-61.

9. Bhora FY, Chen DJ, Detterbeck FC, Asamura H, Falkson C, Filosso PL, et al. The ITMIG/IASLC thymic epithelial tumors staging project: a proposed lymph node map for thymic epithelial tumors in the forthcoming 8th edition of the TNM classification of malignant tumors. J Thorac Oncol. 2014;9(9 Suppl 2): S88-96.

10. Detterbeck FC, Stratton K, Giroux D, Asamura H, Crowley J, Falkson C, et al The IASLC/ITMIG thymic epithelial tumors staging project: proposal for an evidence-based stage classification system for the forthcoming (8th) edition of the TNM classification of malignant tumors. J Thorac Oncol. 2014;9(9 Suppl 2): $: 65-72$

11. Travis WD, Brambilla E, Müller-Hermelink HK, Harris CC, eds. World Health Organization classification of tumours: tumours of the thymus. In: Pathology and Genetics: Tumours of the Lung, Pleura, Thymus and Heart. Berlin: Springer; 2004:146-248.
12. Detterbeck FC, Zeeshan A. Thymoma: current diagnosis and treatment. Chin Med J (Engl). 2013;126:2186-91.

13. Fang W, Yao X, Antonicelli A, Gu Z, Detterbeck F, Vallières E, et al. Comparison of surgical approach and extent of resection for Masaoka-Koga Stage I and II thymic tumors in Europe, North America, and Asia: an International Thymic Malignancy Interest Group retrospective database analysis. Eur J Cardiothorac Surg. 2017;52:26-32.

14. Wang H, Gu Z, Ding J, Tan L, Fu J, Shen Y, et al. Perioperative outcomes and longterm survival in clinically early-stage thymic malignancies: video-assisted thoracoscopic thymectomy versus open approaches. J Thorac Dis. 2016;8:673-9.

Key Words: thymic malignancy, surgery, lymph node metastasis, lymph node dissection

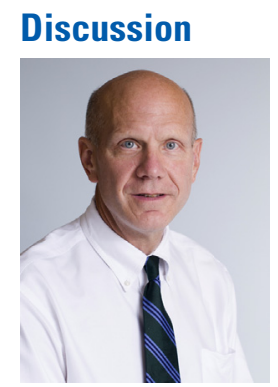

Dr Cameron D. Wright (Boston, Mass). Thank you, Dr Fang, for a very well-presented study that is somewhat provocative, and for providing me with the manuscript. I have two questions. It appears there was some selection bias in dissecting the deep mediastinal nodes, in that those were clearly the worst tumors. Did you have an idea beforehand of the histology or intraoperatively did you find out what the histology was in making that decision between an $\mathrm{N} 1$ versus an N2 nodal dissection?

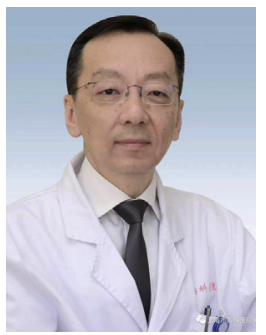

Dr Wentao Fang (Shanghai, China). Thank you. I think it is a very good question. Actually, there was no requirement as to the extent or number of lymph node dissections in this protocol, but we did look into the rate of N2 deep mediastinal dissection in patients with low risk and high risk, and we found a less than $8 \%$ difference between the two groups. Of course, this was not statistically significant, so there is no difference.

Dr Wright. The second question is, moving on, where do we take this study? How do we set it in the context of taking care of our thymic tumor patients? I take your point that we ought to consider dissection for T3, T4, and bad histology lesions with a $15 \%$ to $20 \%$ rate of positive lymph nodes, but what do we do then? I doubt that there is going to be much difference in survival, but importantly right now the standard of care is not to give chemotherapy, but what about giving chemotherapy to these patients?

Dr Fang. I think this is highly relevant to clinical practice. Unfortunately, there is no good evidence that supports postoperative chemotherapy. We do have a protocol at our hospital that would give adjuvant chemotherapy to all patients with lymph node metastasis; however, this is more of an intuitive decision. The Chinese Alliance for Research in Thymomas has also published a retrospective study on the effect of adjuvant chemotherapy for advanced-stage 
thymic tumors; however, even after propensity score matching, we didn't find any beneficial effect of adjuvant chemotherapy. So we really don't know the answer yet.

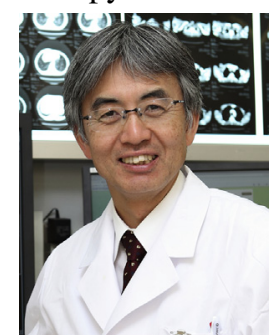

Dr Hiroshi Date (Kyoto, Japan). Vincent, that was an excellent presentation. I would like to know if you have PET scan data before surgery, and if it could pick up the patient with lymph node metastasis?

Dr Fang. That's a good question. At Shanghai Chest, we offer PET scan or nuclear scan for all thymic tumor patients, but it is not a routine practice among all the institutions in this study, because PET scan, as you know, is not covered by insurance in China, and in my experience, its sensitivity of detecting lymph node metastasis in thymic tumors is quite low. It's not that good except for neuroendocrine tumors, which tend to have quite extensive disease involvement.

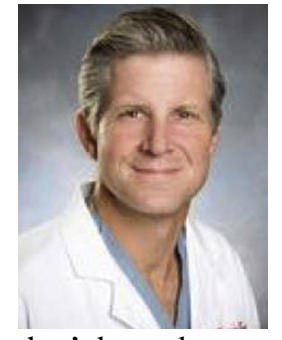

Dr Scott Swanson (Boston, Mass). I enjoyed your paper. It was a great presentation. You may have said this. but I missed it. Do you know how the people with lymph node-positive disease failed? Did they fail locally, distantly? What happened to those patients?

Dr Fang. Thanks for the question. I don't have the exact number, but in my impression, most patients with neuroendocrine tumors tend to have lymph node metastasis and finally distant metastasis; lymph node metastasis always comes first.

Dr Swanson. Do they fail in the chest?

Dr Fang. Most of them would be in the neck.

Dr Swanson. Should they get radiation?

Dr Fang. That's our practice, but I don't know whether if there is any evidence to support that. It is more palliative. 


\section{APPENDIX E1. MEMBERS OF CHINESE ALLIANCE FOR RESEARCH IN THYMOMAS (ChART)}

Yi Shen, Yucheng Wei, Affiliated Hospital of Qingdao University, Qingdao, China; Yin Li, and Guanghui Liang, Affiliated Cancer Hospital of Zhengzhou University, Zhengzhou, China; Keneng Chen and Hao Fu, Beijing Cancer Hospital, Beijing, China; Hezhong Chen and Shihua Yao, Changhai Hospital, Shanghai, China; Youbin Cui and Yanzhong Xin, First Affiliated Hospital of Jilin University, Changchun, China; Renquan Zhang and Ningning Kang, First Hospital of Anhui Medical University, Hefei, China; Lijie Tan, Jianyong Ding, Hao Wang, Gang Chen, and Jie $\mathrm{Wu}$, Zhongshan Hospital, Fudan University, Shanghai, China; Chun Chen and Wei Zheng, Fujian Medical University Union Hospital, Fuzhou, China; Liewen Pang and Fangrui Wang, Huashan Hospital, Fudan University, Shanghai, China; Yangchun Liu and Qing Lin, Jiangxi
People's Hospital, Nanchang, China; Yongyu Liu and Yongkai Wu, Liaoning Cancer Hospital, Shenyang, China; Wentao Fang, Jie Zhang, Yan Shen, Changlu Wang, Lei Zhu and Zhitao Gu, Shanghai Chest Hospital, Shanghai Jiao Tong University, Shanghai, China; Yongtao Han, Lin Peng, Sichuan Cancer Hospital, Chengdu, China; Jianhua Fu and Qianwen Liu, Department of Thoracic Surgery, Guangdong Esophageal Cancer Institute, Sun Yat-sen University Cancer Center, State Key Laboratory of Oncology in South China, Collaborative Innovation Center of Cancer Medicine, Guangzhou, China; Zhentao Yu and Jie Yue, Tianjin Cancer Hospital, Tianjin, China; Peng Zhang and Yuan Chen, Tianjin Medical University General Hospital, Tianjin, China; Yun Wang and Yingcai Geng, West China Hospital, Sichuan University, Chengdu, China; Xinming Zhou and Hongguang Zhao, Zhejiang Cancer Hospital, Hangzhou, China. 